\title{
Neutron electric dipole moment in CP violating BLMSSM
}

\author{
Shu-Min Zhao ${ }^{a *}$, Tai-Fu Feng ${ }^{a \dagger}$, Ben Yan $^{a}$, Hai-Bin Zhang ${ }^{a, b}$, \\ Yin-Jie Zhang ${ }^{a}$, Biao Chen $^{a}$, Xue-Qian $\mathrm{Li}^{c}$ \\ ${ }^{a}$ Department of Physics, Hebei University, Baoding, 071002, China \\ ${ }^{b}$ Department of Physics, Dalian University of Technology, Dalian, 116024, China \\ ${ }^{c}$ Department of Physics, Nankai University, Tianjin, 300071, China
}

(Dated: August 29, 2018)

\begin{abstract}
Considering the $\mathrm{CP}$ violating phases, we analyze the neutron electric dipole moment (EDM) in a CP violating supersymmetric extension of the standard model where baryon and lepton numbers are local gauge symmetries(BLMSSM). The contributions from the one loop diagrams and the Weinberg operators are taken into account. Adopting some assumptions on the relevant parameter space, we give the numerical results analysis. The numerical results for the neutron EDM can reach $1.05 \times 10^{-25}($ e.cm $)$, which is about the experimental upper limit.
\end{abstract}

PACS numbers: 11.30.Er, 12.60.Jv,14.80.Cp

Keywords: CP violating, electric dipole moment, local gauge symmetry

\footnotetext{
* email:smzhao@hbu.edu.cn

$\dagger$ email:fengtf@hbu.edu.cn
} 


\section{INTRODUCTION}

Physicists have been interested in the minimal supersymmetric extension of the standard model (MSSM) [1] for a long time. Considering the matter-antimatter asymmetry in the universe, baryon number (B) should be broken. To explain the neutrino oscillation experiment, neutrino should have tiny mass which can be induced by the seesaw mechanism from the heavy majorana neutrinos. Then lepton number $(\mathrm{L})$ is also expected to be broken. A minimal supersymmetric extension of the SM with local gauged B and L(BLMSSM) is more favorite. With the assumption that B and L are spontaneously broken gauge symmetries around TeV scale, the author [2] examines two extensions of the SM.

$\mathrm{B}$ and L are spontaneously broken near the weak scale in BLMSSM. The light neutrinos get mass from the seesaw mechanism, and proton decay is forbidden[2, 3]. Therefore, a large desert between the electroweak scale and grand unified scale is not necessary. This is the main motivation for the BLMSSM. Many possible signals of the MSSM at the LHC have been studied by the CMS[4] and ATLAS[5] experiments and they set very strong bounds on the gluino and squarks masses with R-parity conservation. However, the predictions and bounds for the collider experiments should be changed with the broken B and L symmetries[2, 6].

Flavour violation in the quark and leptonic sectors is suppressed naturally, because tree level flavor changing neutral currents involving the quarks (charged leptons) are forbidden by the gauge symmetries and particle content[3]. We can look for lepton number violation at the LHC, from the decays of right handed neutrinos [3, 7]. The baryon number violation in the decays of squarks and gauginos [8] can also be detected at the LHC. For example, the baryon number violating decays of gluinos may give rise to channels with multi-tops and multi-bottoms[3].

In BLMSSM, the mass and decays of the lightest CP-even Higgs are investigated, where Yukawa couplings between Higgs doublets and exotic quarks are neglected [2, 3]. Taking into account the Yukawa couplings between Higgs and exotic quarks and ATLAS (CMS) results for the neutral Higgs $\left(m_{h^{0}} \sim 124-126 \mathrm{GeV}\right)[9]$, the lightest CP-even Higgs decays $h^{0} \rightarrow \gamma \gamma$, $h \rightarrow Z Z(W W)$ are investigated in BLMSSM[10].

As is known to all, the magnetic dipole moments(MDMs) [11, 12] and electric dipole 
moments (EDMs) [13] of fermions open a wide window to find new physics beyond the SM. The present experiment upper limit of the neutron EDM is $1.1 \times 10^{-25} \mathrm{e} \cdot \mathrm{cm}$ [14]. In SM, the CP phases of the Cabibbo-Kobayashi-Maskawa (CKM) matrix elements fully induce very tiny neutron EDM, and it is too tiny to be detected by experiment in the near future [15].

In MSSM, many new sources of the CP violation lead to large contributions to the neutron EDM[16, 17] at one loop level, and the theoretical prediction is larger than the present experiment upper bound. To consistent with the experiment data, there are three approaches. The first is fine tuning, making the CP phases sufficiently small, i.e. $\leq 10^{-2}$; The second is that the supersymmetry spectra is very heavy at several TeV. Internal cancellation among the different contributions is the last one, and seems more reasonable[18].

The theoretical analysis of EDMs for the fermions at one-loop level is well studied in MSSM. On other hand, authors obtain the corrections to neutron EDM from the two-loop Barr-Zee type diagrams involving the Higgs bosons[19]. For the neutron EDM, the twoloop gluino corrections and gluonic Weinberg operator induced by two-loop gluino-squark diagrams are carried out in the literature[20].

In BLMSSM, there are many new CP violation sources and new particles. Assuming that the Yukawa couplings between the Higgs and exotic quarks cannot be ignored, we study the neutron EDM from the one loop diagrams and the Weinberg operator contributions in this work. After this introduction, we briefly summarize the main ingredients of the BLMSSM, and show the needed couplings for exotic quarks in section 2. For the neutron EDM, the one loop diagrams' corrections and the Weinberg operator contributions are presented in section 3. In section 4 , we show the numerical results and study the relation between the neutron EDM and the BLMSSM parameters. Our conclusions are summarized in section 5.

\section{SOME COUPLING IN BLMSSM}

Enlarging the SM, the author obtains the BLMSSM [2] with the local gauge group of $S U(3)_{C} \otimes S U(2)_{L} \otimes U(1)_{Y} \otimes U(1)_{B} \otimes U(1)_{L}$. In BLMSSM, to cancel L anomaly, there are the exotic superfields of the new leptons $\hat{L}_{4} \sim\left(1,2,-1 / 2,0, L_{4}\right), \hat{E}_{4}^{c} \sim\left(1,1,1,0,-L_{4}\right)$, $\hat{N}_{4}^{c} \sim\left(1,1,0,0,-L_{4}\right), \hat{L}_{5}^{c} \sim\left(1,2,1 / 2,0,-\left(3+L_{4}\right)\right), \hat{E}_{5} \sim\left(1,1,-1,0,3+L_{4}\right)$, 
$\hat{N}_{5} \sim\left(1,1,0,0,3+L_{4}\right)$. To break lepton number spontaneously, the superfields $\hat{\Phi}_{L} \sim$ $(1,1,0,0,-2)$ and $\hat{\varphi}_{L} \sim(1,1,0,0,2)$ are introduced, and they acquire nonzero vacuum expectation values (VEVs). Higgs mechanism has a solid stone because of the detection of the lightest CP even Higgs at LHC[9].

In the same way, the model includes the new quarks $\hat{Q}_{4} \sim\left(3,2,1 / 6, B_{4}, 0\right), \hat{U}_{4}^{c} \sim$ $\left(\overline{3}, 1,-2 / 3,-B_{4}, 0\right), \hat{D}_{4}^{c} \sim\left(\overline{3}, 1,1 / 3,-B_{4}, 0\right), \hat{Q}_{5}^{c} \sim\left(\overline{3}, 2,-1 / 6,-\left(1+B_{4}\right), 0\right)$, $\hat{U}_{5} \sim\left(3,1,2 / 3,1+B_{4}, 0\right), \hat{D}_{5} \sim\left(3,1,-1 / 3,1+B_{4}, 0\right)$, to cancel the B anomaly. $\hat{\Phi}_{B} \sim(1,1,0,1,0)$ and $\hat{\varphi}_{B} \sim(1,1,0,-1,0)$ are the 'brand new' Higgs superfields. With nonzero vacuum expectation values (VEVs) they break baryon number spontaneously. The exotic quarks are very heavy, because of the nonzero VEVs of $\Phi_{B}$ and $\varphi_{B}$. Therefore, the BLMSSM also introduces the superfields $\hat{X} \sim\left(1,1,0,2 / 3+B_{4}, 0\right), \hat{X}^{\prime} \sim(1,1,0,-(2 / 3+$ $\left.\left.B_{4}\right), 0\right)$ to make the unstable exotic quarks. The lightest superfields $\mathrm{X}$ can be a candidate for dark matter.

In BLMSSM, the superpotential reads as follows

$$
\mathcal{W}_{B L M S S M}=\mathcal{W}_{M S S M}+\mathcal{W}_{B}+\mathcal{W}_{L}+\mathcal{W}_{X}
$$

with $\mathcal{W}_{\text {MSSM }}$ representing the superpotential of the MSSM. The concrete forms of $\mathcal{W}_{B}, \mathcal{W}_{L}$ and $\mathcal{W}_{X}$ are

$$
\begin{aligned}
\mathcal{W}_{B}= & \lambda_{Q} \hat{Q}_{4} \hat{Q}_{5}^{c} \hat{\Phi}_{B}+\lambda_{U} \hat{U}_{4}^{c} \hat{U}_{5} \hat{\varphi}_{B}+\lambda_{D} \hat{D}_{4}^{c} \hat{D}_{5} \hat{\varphi}_{B}+\mu_{B} \hat{\Phi}_{B} \hat{\varphi}_{B} \\
& +Y_{u_{4}} \hat{Q}_{4} \hat{H}_{u} \hat{U}_{4}^{c}+Y_{d_{4}} \hat{Q}_{4} \hat{H}_{d} \hat{D}_{4}^{c}+Y_{u_{5}} \hat{Q}_{5}^{c} \hat{H}_{d} \hat{U}_{5}+Y_{d_{5}} \hat{Q}_{5}^{c} \hat{H}_{u} \hat{D}_{5}, \\
\mathcal{W}_{L}= & Y_{e_{4}} \hat{L}_{4} \hat{H}_{d} \hat{E}_{4}^{c}+Y_{\nu_{4}} \hat{L}_{4} \hat{H}_{u} \hat{N}_{4}^{c}+Y_{e_{5}} \hat{L}_{5}^{c} \hat{H}_{u} \hat{E}_{5}+Y_{\nu_{5}} \hat{L}_{5}^{c} \hat{H}_{d} \hat{N}_{5} \\
& +Y_{\nu} \hat{L} \hat{H}_{u} \hat{N}^{c}+\lambda_{N^{c}} \hat{N}^{c} \hat{N}^{c} \hat{\varphi}_{L}+\mu_{L} \hat{\Phi}_{L} \hat{\varphi}_{L}, \\
\mathcal{W}_{X}= & \lambda_{1} \hat{Q} \hat{Q}_{5}^{c} \hat{X}+\lambda_{2} \hat{U}^{c} \hat{U}_{5} \hat{X}^{\prime}+\lambda_{3} \hat{D}^{c} \hat{D}_{5} \hat{X}^{\prime}+\mu_{X} \hat{X} \hat{X}^{\prime} .
\end{aligned}
$$

The soft breaking terms $\mathcal{L}_{\text {soft }}$ of the BLMSSM can be found in our previous work [10].

To make the local gauge symmetry $S U(2)_{L} \otimes U(1)_{Y} \otimes U(1)_{B} \otimes U(1)_{L}$ broken down to the electromagnetic symmetry $U(1)_{e}$, the $S U(2)_{L}$ singlets $\Phi_{B}, \varphi_{B}, \Phi_{L}, \varphi_{L}$ and the $S U(2)_{L}$ doublets $H_{u}, H_{d}$ should obtain nonzero VEVs $v_{B}, \bar{v}_{B}, v_{L}, \bar{v}_{L}$ and $v_{u}, v_{d}$ respectively.

$$
H_{u}=\left(\begin{array}{c}
H_{u}^{+} \\
\frac{1}{\sqrt{2}}\left(v_{u}+H_{u}^{0}+i P_{u}^{0}\right)
\end{array}\right), \quad H_{d}=\left(\begin{array}{c}
\frac{1}{\sqrt{2}}\left(v_{d}+H_{d}^{0}+i P_{d}^{0}\right) \\
H_{d}^{-}
\end{array}\right),
$$




$$
\begin{array}{ll}
\Phi_{B}=\frac{1}{\sqrt{2}}\left(v_{B}+\Phi_{B}^{0}+i P_{B}^{0}\right), & \varphi_{B}=\frac{1}{\sqrt{2}}\left(\bar{v}_{B}+\varphi_{B}^{0}+i \bar{P}_{B}^{0}\right), \\
\Phi_{L}=\frac{1}{\sqrt{2}}\left(v_{L}+\Phi_{L}^{0}+i P_{L}^{0}\right), & \varphi_{L}=\frac{1}{\sqrt{2}}\left(\bar{v}_{L}+\varphi_{L}^{0}+i \bar{P}_{L}^{0}\right),
\end{array}
$$

In Ref.[10], the mass matrixes of Higgs, exotic quarks and exotic scalar quarks are obtained. In the mass basis, assuming $\mathrm{CP}$ conservation in exotic quark sector, we show the couplings between the neutral Higgs and charged $(2 / 3,-1 / 3)$ exotic quarks. Here we investigate the $\mathrm{CP}$ violating effect and modify our previous results.

To save space, we define $H_{\alpha}^{0}(\alpha=1,2,3, \ldots 8)=\left(h^{0}, H^{0}, A^{0}, G^{0}, h_{B}^{0}, H_{B}^{0}, A_{B}^{0}, G_{B}^{0}\right)$.

$$
\begin{aligned}
\mathcal{L}_{H^{0} q^{\prime} q^{\prime}}= & \sum_{\alpha=1}^{8} \sum_{i, j=1}^{2}\left\{\left(\mathcal{N}_{H_{\alpha}^{0}}^{L}\right)_{i j} H_{\alpha}^{0} \bar{t}_{i+3} P_{L} t_{j+3}+\left(\mathcal{N}_{H_{\alpha}^{0}}^{R}\right)_{i j} H_{\alpha}^{0} \bar{t}_{i+3} P_{R} t_{j+3}\right. \\
& \left.+\left(\mathcal{K}_{H_{\alpha}^{0}}^{L}\right)_{i j} H_{\alpha}^{0} \bar{b}_{i+3} P_{L} b_{j+3}+\left(\mathcal{K}_{H_{\alpha}^{0}}^{R}\right)_{i j} H_{\alpha}^{0} \bar{b}_{i+3} P_{R} b_{j+3}\right\},
\end{aligned}
$$

where $t_{i+3}, b_{i+3}(i=1,2)$ are the exotic quark fields, the coupling contents $\left(\mathcal{N}_{H_{\alpha}^{0}}^{L}\right)_{i j},\left(\mathcal{N}_{H_{\alpha}^{0}}^{L}\right)_{i j},\left(\mathcal{N}_{H_{\alpha}^{0}}^{L}\right)_{i j},\left(\mathcal{N}_{H_{\alpha}^{0}}^{L}\right)_{i j}$ are collected in the appendix.

The couplings between charged Higgs and exotic quarks are

$$
\begin{aligned}
\mathcal{L}_{H^{+} t^{\prime} b^{\prime}}= & \sum_{i, j=1}^{2}\left[\left(\mathcal{N}_{H^{+}}^{L}\right)_{i j} H^{+} \bar{t}_{i+3} P_{L} b_{j+3}+\left(\mathcal{N}_{H^{+}}^{R}\right)_{i j} H^{+} \bar{t}_{i+3} P_{R} b_{j+3}\right] \\
& +\sum_{i, j=1}^{2}\left[\left(\mathcal{N}_{G^{+}}^{L}\right)_{i j} G^{+} \bar{t}_{i+3} P_{L} b_{j+3}+\left(\mathcal{N}_{G^{+}}^{R}\right)_{i j} G^{+} \bar{t}_{i+3} P_{R} b_{j+3}\right]+\text { h.c. }
\end{aligned}
$$

with the coupling constants

$$
\begin{aligned}
& \left(\mathcal{N}_{H^{+}}^{L}\right)_{i j}=-\left(Y_{u_{4}}\left(W_{t}^{\dagger}\right)_{i 2}\left(U_{b}\right)_{1 j}+Y_{d_{5}}\left(W_{t}^{\dagger}\right)_{i 1}\left(U_{b}\right)_{2 j}\right) \cos \beta, \\
& \left(\mathcal{N}_{H^{+}}^{R}\right)_{i j}=\left(Y_{d_{4}}^{*}\left(U_{t}^{\dagger}\right)_{i 1}\left(W_{b}\right)_{2 j}+Y_{u_{5}}^{*}\left(U_{t}^{\dagger}\right)_{i 2}\left(W_{b}\right)_{1 j}\right) \sin \beta, \\
& \left(\mathcal{N}_{G^{+}}^{L}\right)_{i j}=-\left(Y_{u_{4}}\left(W_{t}^{\dagger}\right)_{i 2}\left(U_{b}\right)_{1 j}+Y_{d_{5}}\left(W_{t}^{\dagger}\right)_{i 1}\left(U_{b}\right)_{2 j}\right) \sin \beta, \\
& \left(\mathcal{N}_{G^{+}}^{R}\right)_{i j}=-\left(Y_{d_{4}}^{*}\left(U_{t}^{\dagger}\right)_{i 1}\left(W_{b}\right)_{2 j}+Y_{u_{5}}^{*}\left(U_{t}^{\dagger}\right)_{i 2}\left(W_{b}\right)_{1 j}\right) \cos \beta .
\end{aligned}
$$

Here, $\tan \beta=v_{u} / v_{d}$. $W_{t}$ and $U_{t}$ are the matrixes to diagonalize the mass matrix of the up type exotic quarks. While, $W_{b}$ and $U_{b}$ are the matrixes to diagonalize the mass matrix of the exotic quarks with charge $-1 / 3 . A_{t_{i+3}}, A_{d_{i+3}}(i=1,2)$ are the same with those in Ref.[10]. We also derive the couplings between photon (gluon) and exotic quarks.

$$
\mathcal{L}_{\gamma q^{\prime} q^{\prime}}=-\frac{2 e}{3} \sum_{i=1}^{2} \bar{t}_{i+3} \gamma^{\mu} t_{i+3} F_{\mu}+\frac{e}{3} \sum_{i=1}^{2} \bar{b}_{i+3} \gamma^{\mu} b_{i+3} F_{\mu}
$$




$$
\mathcal{L}_{g q^{\prime} q^{\prime}}=-g_{3} \sum_{i=1}^{2} \bar{t}_{i+3} T^{a} \gamma^{\mu} t_{i+3} G_{\mu}^{a}-g_{3} \sum_{i=1}^{2} \bar{b}_{i+3} T^{a} \gamma^{\mu} b_{i+3} G_{\mu}^{a},
$$

where $F_{\mu}$ is electromagnetic field, $G_{\mu}^{a}$ is gluon field, $T^{a}(a=1, \cdots, 8)$ denote the generators of the strong $S U(3)$ gauge group.

The couplings of gluino, exotic quark and exotic scalar quark have relation with Weinberg operator, and are written in the following form

$$
\begin{aligned}
& \mathcal{L}_{\Lambda t^{\prime} \tilde{\mathcal{U}}}=\sqrt{2} g_{3} T^{a} \sum_{i=1}^{4} \sum_{j=1}^{2}\left\{\left(\mathcal{N}_{\tilde{\mathcal{U}}}^{L}\right)_{i j} \tilde{\mathcal{U}}_{i}^{*} \bar{\Lambda}_{G} P_{L} t_{j+3}+\left(\mathcal{N}_{\tilde{\mathcal{U}}}^{R}\right)_{i j} \tilde{\mathcal{U}}_{i}^{*} \bar{\Lambda}_{G} P_{R} t_{j+3}\right\}+\text { h.c. } \\
& \mathcal{L}_{\Lambda b^{\prime} \tilde{\mathcal{D}}}=\sqrt{2} g_{3} T^{a} \sum_{i=1}^{4} \sum_{j=1}^{2}\left\{\left(\mathcal{N}_{\tilde{\mathcal{D}}}^{L}\right)_{i j} \tilde{\mathcal{D}}_{i}^{*} \bar{\Lambda}_{G} P_{L} b_{j+3}+\left(\mathcal{N}_{\tilde{\mathcal{D}}}^{R}\right)_{i j} \tilde{\mathcal{D}}_{i}^{*} \bar{\Lambda}_{G} P_{R} b_{j+3}\right\}+\text { h.c. }
\end{aligned}
$$

where $\Lambda_{G}$ is gluino field. $\tilde{\mathcal{U}}_{i}, \tilde{\mathcal{D}}_{i}(i=1,2,3,4)$ are exotic scalar quarks with charge $2 / 3$ and $-1 / 3$ respectively. The concrete forms of the coupling constants are

$$
\begin{array}{ll}
\left(\mathcal{N}_{\tilde{\mathcal{U}}}^{L}\right)_{i j}=U_{i 4}^{\dagger} U_{2 j}^{t}-U_{i 1}^{\dagger} U_{1 j}^{t}, & \left(\mathcal{N}_{\tilde{\mathcal{U}}}^{R}\right)_{i j}=U_{i 2}^{\dagger} W_{2 j}^{t}-U_{i 3}^{\dagger} W_{1 j}^{t}, \\
\left(\mathcal{N}_{\tilde{\mathcal{D}}}^{L}\right)_{i j}=D_{i 4}^{\dagger} U_{2 j}^{b}-D_{i 1}^{\dagger} U_{1 j}^{b}, & \left(\mathcal{N}_{\tilde{\mathcal{D}}}^{R}\right)_{i j}=D_{i 2}^{\dagger} W_{2 j}^{b}-D_{i 3}^{\dagger} W_{1 j}^{b} .
\end{array}
$$

Using the scalar potential and the soft breaking terms, we write the mass squared matrix for superfields X.

$$
-\mathcal{L}_{X}=\left(\begin{array}{ll}
X^{*} & X^{\prime}
\end{array}\right)\left(\begin{array}{cc}
\left|\mu_{X}\right|^{2}+S_{X} & -\mu_{X}^{*} B_{X}^{*} \\
-\mu_{X} B_{X} & \left|\mu_{X}\right|^{2}-S_{X}
\end{array}\right)\left(\begin{array}{c}
X \\
X^{\prime *}
\end{array}\right),
$$

with $S_{X}=\frac{g_{B}^{2}}{2}\left(\frac{2}{3}+B_{4}\right)\left(v_{B}^{2}-\bar{v}_{B}^{2}\right)$. Adopting the unitary transformation,

$$
\left(\begin{array}{c}
X_{1} \\
X_{2}
\end{array}\right)=Z_{X}^{\dagger}\left(\begin{array}{c}
X \\
X^{* *}
\end{array}\right)
$$

the mass squared matrix for the superfields $\mathrm{X}$ are diagonalized.

$$
Z_{X}^{\dagger}\left(\begin{array}{cc}
\left|\mu_{X}\right|^{2}+S_{X} & -\mu_{X}^{*} B_{X}^{*} \\
-\mu_{X} B_{X} & \left|\mu_{X}\right|^{2}-S_{X}
\end{array}\right) Z_{X}=\left(\begin{array}{cc}
m_{X 1}^{2} & 0 \\
0 & m_{X 2}^{2}
\end{array}\right) .
$$

In mass basis, we obtain the couplings of quark-exotic quark and the superfields X.

$$
\begin{aligned}
& \mathcal{L}_{X t^{\prime} u}=\sum_{i, j=1}^{2}\left(\left(\mathcal{N}_{t^{\prime}}^{L}\right)_{i j} X_{j} \bar{t}_{i+3} P_{L} u^{I}+\left(\mathcal{N}_{t^{\prime}}^{R}\right)_{i j} X_{j} \bar{t}_{i+3} P_{R} u^{I}\right)+\text { h.c. } \\
& \mathcal{L}_{X b^{\prime} d}=\sum_{i, j=1}^{2}\left(\left(\mathcal{N}_{b^{\prime}}^{L}\right)_{i j} X_{j} \bar{b}_{i+3} P_{L} d^{I}+\left(\mathcal{N}_{b^{\prime}}^{R}\right)_{i j} X_{j} \bar{b}_{i+3} P_{R} d^{I}\right)+\text { h.c. }
\end{aligned}
$$


where the coupling constants are

$$
\begin{array}{ll}
\left(\mathcal{N}_{t^{\prime}}^{L}\right)_{i j}=-\lambda_{1}\left(W_{t}^{\dagger}\right)_{i 1}\left(Z_{X}\right)_{1 j}, & \left(\mathcal{N}_{t^{\prime}}^{R}\right)_{i j}=-\lambda_{2}^{*}\left(U_{t}^{\dagger}\right)_{i 2}\left(Z_{X}\right)_{2 j}, \\
\left(\mathcal{N}_{b^{\prime}}^{L}\right)_{i j}=\lambda_{1}\left(W_{b}^{\dagger}\right)_{i 1}\left(Z_{X}\right)_{1 j}, & \left(\mathcal{N}_{b^{\prime}}^{R}\right)_{i j}=-\lambda_{3}^{*}\left(U_{b}^{\dagger}\right)_{i 2}\left(Z_{X}\right)_{2 j} .
\end{array}
$$

\section{THE CORRECTIONS TO THE NEUTRON EDM IN BLMSSM}

The $\operatorname{EDM} d_{f}$ of the fermion can be obtained from the effective Lagrangian[20]

$$
\mathcal{L}_{E D M}=-\frac{i}{2} d_{f} \bar{f} \sigma^{\mu \nu} \gamma_{5} f F_{\mu \nu}
$$

where $F_{\mu \nu}$ represents the electromagnetic field strength, $f$ is a fermion field. This effective Lagrangian violates $\mathrm{CP}$ conservation obviously, and cannot be present at tree level in the fundamental interactions. However in the CP violating electroweak theory, it can be obtained at least at one loop level.

Because neutron is composed of quarks and gluons, the chromoelectric dipole moment (CEDM) $\bar{f} T^{a} \sigma^{\mu \nu} \gamma_{5} f G_{\mu \nu}^{a}$ of quarks can also give contributions to the neutron EDM. $G_{\mu \nu}^{a}$ represents the gluon field strength. Weinberg has discovered a dimension-6 operator $f_{a b c} G_{\mu \rho}^{a} G_{\nu}^{b \rho} G_{\lambda \sigma}^{c} \epsilon^{\mu \nu \lambda \sigma}$ which violates CP conservation and it makes a very large contribution to the neutron $\mathrm{EDM}[21]$.

The effective method is convenient to describe the CP violation operators at loop level. At matching scale, we can integrate out the heavy particles in the loop and obtain the resulting effective Lagrangian with a full set of $\mathrm{CP}$ violation operators. Here, we just show the effective Lagrangian containing operators relevant to the neutron EDM.

$$
\begin{aligned}
& \mathcal{L}_{\text {eff }}=\sum_{i}^{5} C_{i}(\Lambda) \mathcal{O}_{i}(\Lambda) \\
& \mathcal{O}_{1}=\bar{q} \sigma^{\mu \nu} P_{L} q F_{\mu \nu}, \quad \mathcal{O}_{2}=\bar{q} \sigma^{\mu \nu} P_{R} q F_{\mu \nu}, \quad \mathcal{O}_{3}=\bar{q} T^{a} \sigma^{\mu \nu} P_{L} q G_{\mu \nu}^{a}, \\
& \mathcal{O}_{4}=\bar{q} T^{a} \sigma^{\mu \nu} P_{R} q G_{\mu \nu}^{a}, \mathcal{O}_{5}=-\frac{1}{6} f_{a b c} G_{\mu \rho}^{a} G_{\nu}^{b \rho} G_{\lambda \sigma}^{c} \epsilon^{\mu \nu \lambda \sigma} .
\end{aligned}
$$

where $\Lambda$ is the energy scale, at which the Wilson coefficients $C_{i}(\Lambda)$ are evaluated. 


\section{A. The corrections to neutron EDM in MSSM}

In SUSY, the one-loop supersymmetric corrections to the quark EDMs and CEDMs are obtained[20]. It can be divided into three types according to the quark self-energy diagrams. The internal line particles are chargino and squark (neutralino and squark, gluino and squark). The needed triangle diagrams are obtained by attaching a photon or gluon on the internal lines of the quark selfenergy diagrams in all possible ways. Calculating these diagrams one can obtain the effective Lagrangian contributing to the quark EDMs and CEDMs. Before we show the BLMSSM corrections, we present the one loop MSSM contributions to the quark EDMs and CEDMs, which can be obtained in the previous works. The needed one loop self energy diagrams are shown in Fig.(11).
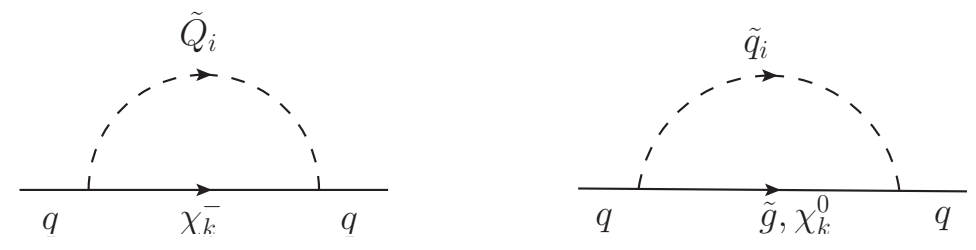

FIG. 1: The MSSM one loop self energy diagrams from which the corresponding triangle diagrams are obtained by attaching a photon or a gluon in all possible ways.

The one-loop neutralino-squark corrections to the quark EDMs and CEDMs are

$$
\begin{aligned}
& d_{\chi_{k}^{0}(1)}^{\gamma}=e_{q} \frac{e \alpha}{16 \pi s_{\mathrm{w}}^{2} c_{\mathrm{w}}^{2}} \sum_{i=1}^{2} \sum_{k=1}^{4} \operatorname{Im}\left(\left(A_{N}^{q}\right)_{k, i}\left(B_{N}^{q}\right)_{i, k}^{\dagger}\right) \frac{m_{\chi_{k}^{0}}}{m_{\tilde{q}_{i}}^{2}} B\left(\frac{m_{k}^{2}}{m_{\tilde{q}_{i}}^{2}}\right), \\
& d_{\chi_{k}^{0}(1)}^{g}=\frac{g_{3} \alpha_{s}}{64 \pi s_{\mathrm{w}}^{2} c_{\mathrm{w}}^{2}} \sum_{i=1}^{2} \sum_{k=1}^{4} \operatorname{Im}\left(\left(A_{N}^{q}\right)_{k, i}\left(B_{N}^{q}\right)_{i, k}^{\dagger}\right) \frac{m_{\chi_{k}^{0}}}{m_{\tilde{q}_{i}}^{2}} B\left(\frac{m_{k}^{2}}{m_{\tilde{q}_{i}}^{2}}\right),
\end{aligned}
$$

Here $e_{u}=2 / 3, e_{d}=-1 / 3, \alpha=e^{2} /(4 \pi), \alpha_{s}=g_{3}^{2} /(4 \pi), s_{W}=\sin \theta_{W}, c_{W}=\cos \theta_{W}, \theta_{W}$ is the Weinberg angle. $m_{\tilde{q}_{i}}(i=1,2)$ are the scalar quarks masses and $m_{\chi_{k}^{0}}(k=1,2,3,4)$ denote the eigenvalues of neutralino mass matrix. We define the one loop function $B(r)$ as $B(r)=\left[2(r-1)^{2}\right]^{-1}[1+r+2 r \ln r /(r-1)]$. The concrete forms of the coupling constants $\left(A_{N}^{u}\right)_{k, i},\left(B_{N}^{u}\right)_{k, i},\left(A_{N}^{d}\right)_{k, i},\left(B_{N}^{d}\right)_{k, i}$ can be found in Ref.[20].

The contributions from the one loop gluino-squark diagrams are written as

$$
d_{\tilde{g}(1)}^{\gamma}=-\frac{2}{3 \pi} e_{q} e \alpha_{s} \sum_{i=1}^{2} \operatorname{Im}\left(\left(\mathcal{Z}_{\tilde{q}}\right)_{2, i}\left(\mathcal{Z}_{\tilde{q}}^{\dagger}\right)_{i, 1} e^{-i \theta_{3}}\right) \frac{\left|m_{\tilde{g}}\right|}{m_{\tilde{q}_{i}}^{2}} B\left(\frac{\left|m_{\tilde{g}}\right|^{2}}{m_{\tilde{q}_{i}}^{2}}\right),
$$




$$
d_{\tilde{g}(1)}^{g}=\frac{g_{3} \alpha_{s}}{4 \pi} \sum_{i=1}^{2} \operatorname{Im}\left(\left(\mathcal{Z}_{\tilde{q}}\right)_{2, i}\left(\mathcal{Z}_{\tilde{q}}^{\dagger}\right)_{i, 1} e^{-i \theta_{3}}\right) \frac{\left|m_{\tilde{g}}\right|}{m_{\tilde{q}_{i}}^{2}} C\left(\frac{\left|m_{\tilde{g}}\right|^{2}}{m_{\tilde{q}_{i}}^{2}}\right)
$$

with $\theta_{3}$ denoting the phase of the gluino mass $m_{\tilde{g}}$ and the loop function $C(r)=[6(r-$ $\left.1)^{2}\right]^{-1}[10 r-26-(2 r-18) \ln r /(r-1)] . \mathcal{Z}_{\tilde{q}}$ are the mixing matrices of the squarks, which diagonalize the mass squared matrixes of squarks $\mathcal{Z}_{\tilde{q}}^{\dagger} \mathbf{m}_{\tilde{q}}^{2} \mathcal{Z}_{\tilde{q}}=\operatorname{diag}\left(m_{\tilde{q}_{1}}^{2}, m_{\tilde{q}_{2}}^{2}\right)$.

We give the chargino-squark contributions here

$$
\begin{aligned}
d_{\chi_{k}^{ \pm}(1)}^{\gamma}= & \frac{e \alpha}{4 \pi s_{\mathrm{w}}^{2}} V_{q Q}^{\dagger} V_{Q q} \sum_{i, k} \operatorname{Im}\left(\left(A_{C}^{Q}\right)_{k, i}\left(B_{C}^{Q}\right)_{i, k}^{\dagger}\right) \frac{m_{k}^{ \pm}}{m_{\tilde{Q}_{i}}^{2}} \\
& \times\left[e_{Q} B\left(\frac{m^{2}}{m_{\tilde{Q}_{i}}^{2}}\right)+\left(e_{q}-e_{Q}\right) A\left(\frac{m^{2}}{m_{\tilde{Q}_{i}}^{2}}\right)\right], \\
d_{\chi_{k}^{ \pm}(1)}^{g} & =\frac{g_{3} \alpha}{4 \pi s_{\mathrm{w}}^{2}} V_{q Q}^{\dagger} V_{Q q} \sum_{i, k} \operatorname{Im}\left(\left(A_{C}^{Q}\right)_{k, i}\left(B_{C}^{Q}\right)_{i, k}^{\dagger}\right) \frac{\chi_{k}^{ \pm}}{m_{\tilde{Q}_{i}}^{2}} B\left(\frac{m^{2}}{m_{\tilde{Q}_{i}}^{2}}\right),
\end{aligned}
$$

where $m_{\chi_{k}^{ \pm}}(k=1,2)$ denote the chargino masses, $V$ is the CKM matrix, and the concrete form of $A(r)$ is $A(r)=\left[2(1-r)^{2}\right]^{-1}[3-r+2 \ln r /(1-r)]$. One can obtain the concrete forms of $\left(A_{C}^{d}\right)_{k, i},\left(B_{C}^{d}\right)_{k, i},\left(A_{C}^{u}\right)_{k, i},\left(B_{C}^{u}\right)_{k, i}$ in the literature[20].

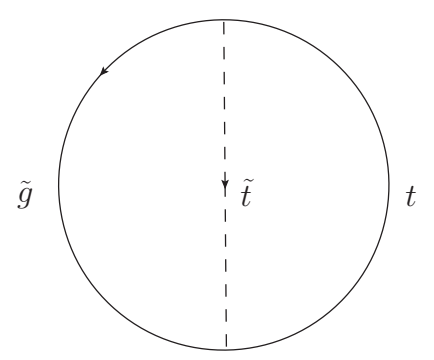

FIG. 2: The MSSM two loop diagram contributing to the Weinberg operator by attaching three gluons in all possible ways.

In MSSM, the two-loop "gluino-squark" diagram shown in Fig.(2) gives contributions to the gluonic Weinberg operator, whose Wilson coefficient is [20, 21]

$$
C_{5}^{S U S Y}=-3 \alpha_{s} m_{t}\left(\frac{g_{3}}{4 \pi}\right)^{3} \operatorname{Im}\left(\left(\mathcal{Z}_{\tilde{t}}\right)_{2,2}\left(\mathcal{Z}_{\tilde{t}}\right)_{2,1}^{\dagger}\right) \frac{m_{\tilde{t}_{1}}^{2}-m_{\tilde{t}_{2}}^{2}}{\left|m_{\tilde{g}}\right|^{5}} H\left(\frac{m_{\tilde{t}_{1}}^{2}}{\left|m_{\tilde{g}}\right|^{2}}, \frac{m_{\tilde{t}_{2}}^{2}}{\left|m_{\tilde{g}}\right|^{2}}, \frac{m_{t}^{2}}{\left|m_{\tilde{g}}\right|^{2}}\right)
$$

Here, the $H$ function can be found in [21]. $\mathcal{Z}_{\tilde{t}}$ is the matrix to diagonalize the mass squared matrix of stop. Up to now, in MSSM the dominant contributions to the neutron EDM are complete. 


\section{B. The corrections to the neutron EDM from superfields in BLMSSM}

In BLMSSM, the quark, exotic quark and superfields $\mathrm{X}$ have a coupling at tree level in Eq.(13) to avoid stability for the exotic quarks. Therefore, there are one loop triangle diagrams obtained by attaching a photon or a gluon in all possible ways on the self energy diagram in Fig.(3). Then we get the quark EDMs and CEDMs.

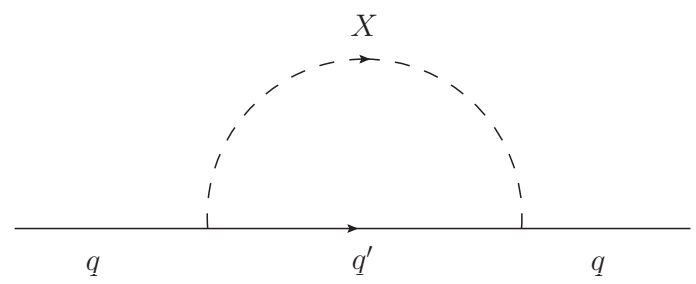

FIG. 3: The one loop selfenergy diagram from which the corresponding triangle diagrams are obtained by attaching a photon or a gluon in all possible ways.

$$
\begin{aligned}
& d_{X_{j}}^{\gamma}=-\frac{e_{q} e}{64 \pi^{2}} \sum_{i, j=1}^{2} \frac{m_{q_{i+3}}}{m_{X_{j}}^{2}} \operatorname{Im}\left(\left(\mathcal{N}_{q^{\prime}}^{L}\right)_{i j}\left(\mathcal{N}_{q^{\prime}}^{R}\right)_{j i}^{\dagger}\right) A\left(\frac{m_{q_{i+3}}^{2}}{m_{X_{j}}^{2}}\right), \\
& d_{X_{j}}^{g}=-\frac{g_{3}}{64 \pi^{2}} \sum_{i, j=1}^{2} \frac{m_{q_{i+3}}}{m_{X_{j}}^{2}} \operatorname{Im}\left(\left(\mathcal{N}_{q^{\prime}}^{L}\right)_{i j}\left(\mathcal{N}_{q^{\prime}}^{R}\right)_{j i}^{\dagger}\right) A\left(\frac{m_{q_{i+3}}^{2}}{m_{X_{j}}^{2}}\right),
\end{aligned}
$$

where $q=t(b), q^{\prime}=t^{\prime}\left(b^{\prime}\right), e_{t^{\prime}}=2 / 3, e_{b^{\prime}}=-1 / 3, m_{X_{j}}(j=1,2)$ denote the masses of the superfields X, $m_{t_{i+3}}(i=1,2)$ represent the masses of the up type exotic quarks, $m_{b_{i+3}}(i=$ $1,2)$ are the masses of the down type exotic quarks.

The Weinberg operator comes from the two loop gluon diagrams. In this model, there are four exotic quarks and eight exotic scalar quarks, which leads to a lot of diagrams contributing to the Wilson coefficient $C_{5}(\Lambda)$ of the Weinberg operator. These two loop diagrams are drawn in Fig.(4). The two loop "gluino-exotic quark-exotic scalar quark" diagrams have corrections to the Wilson coefficient of the purely gluonic Weinberg operator, and the results are presented here.

$$
\begin{aligned}
C_{5}^{\Lambda t^{\prime} \tilde{\mathcal{U}}}= & 3 \alpha_{s}\left(\frac{g_{3}}{4 \pi}\right)^{3} \sum_{i=1}^{2} m_{t_{i+3}}\left[\operatorname{Im}\left(\left(\mathcal{N}_{\tilde{\mathcal{U}}}^{L}\right)_{i 2}^{\dagger}\left(\mathcal{N}_{\tilde{\mathcal{U}}}^{R}\right)_{2 i}\right) \frac{m_{\tilde{\mathcal{U}}_{1}}^{2}-m_{\tilde{\mathcal{U}}_{2}}^{2}}{\left|m_{\tilde{g}}\right|^{5}} H\left(\frac{m_{\tilde{\mathcal{U}}_{1}}^{2}}{\left|m_{\tilde{g}}\right|^{2}}, \frac{m_{\tilde{\mathcal{U}}_{2}}^{2}}{\left|m_{\tilde{g}}\right|^{2}}, \frac{m_{t_{i+3}}^{2}}{\left|m_{\tilde{g}}\right|^{2}}\right)\right. \\
& \left.+\operatorname{Im}\left(\left(\mathcal{N}_{\tilde{\tilde{\mathcal{U}}}}^{L}\right)_{i 4}^{\dagger}\left(\mathcal{N}_{\tilde{\tilde{\mathcal{U}}}}^{R}\right)_{4 i}\right) \frac{m_{\tilde{\mathcal{U}}_{3}}^{2}-m_{\tilde{\mathcal{U}}_{4}}^{2}}{\left|m_{\tilde{g}}\right|^{5}} H\left(\frac{m_{\tilde{\mathcal{U}}_{3}}^{2}}{\left|m_{\tilde{g}}\right|^{2}}, \frac{m_{\tilde{\mathcal{U}}_{4}}^{2}}{\left|m_{\tilde{g}}\right|^{2}}, \frac{m_{t_{i+3}}^{2}}{\left|m_{\tilde{g}}\right|^{2}}\right)\right]
\end{aligned}
$$




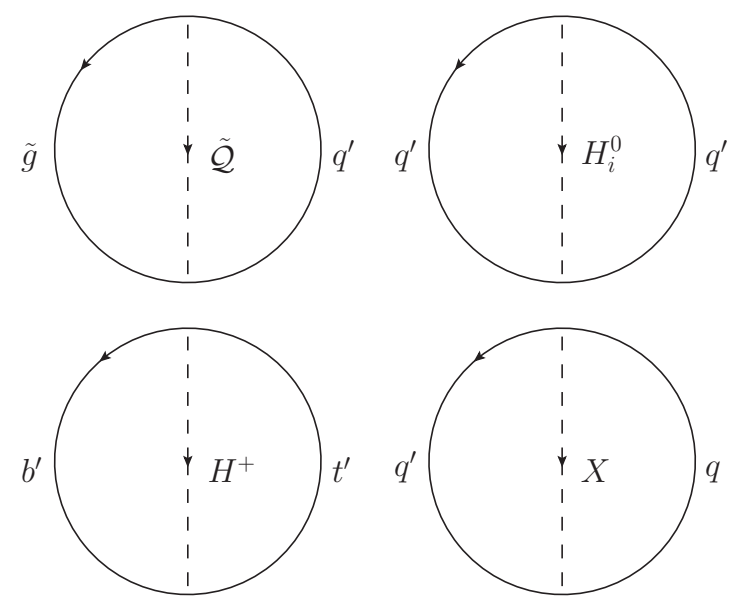

FIG. 4: The BLMSSM two loop diagrams contributing to the Weinberg operator by attaching three gluons in all possible ways.

$$
\begin{aligned}
C_{5}^{\Lambda b^{\prime} \tilde{\mathcal{D}}}= & 3 \alpha_{s}\left(\frac{g_{3}}{4 \pi}\right)^{3} \sum_{i=1}^{2} m_{b_{i+3}}\left[\operatorname{Im}\left(\left(\mathcal{N}_{\tilde{\mathcal{D}}}^{L}\right)_{i 2}^{\dagger}\left(\mathcal{N}_{\tilde{\mathcal{D}}}^{R}\right)_{2 i}\right) \frac{m_{\tilde{\mathcal{D}}_{1}}^{2}-m_{\tilde{\mathcal{D}}_{2}}^{2}}{\left|m_{\tilde{g}}\right|^{5}} H\left(\frac{m_{\tilde{\mathcal{D}}_{1}}^{2}}{\left|m_{\tilde{g}}\right|^{2}}, \frac{m_{\tilde{\mathcal{D}}_{2}}^{2}}{\left|m_{\tilde{g}}\right|^{2}}, \frac{m_{b_{i+3}}^{2}}{\left|m_{\tilde{g}}\right|^{2}}\right)\right. \\
& \left.+\operatorname{Im}\left(\left(\mathcal{N}_{\tilde{\mathcal{D}}}^{L}\right)_{i 4}^{\dagger}\left(\mathcal{N}_{\tilde{\tilde{\mathcal{D}}}}^{R}\right)_{4 i}\right) \frac{m_{\tilde{\mathcal{D}}_{3}}^{2}-m_{\tilde{\mathcal{D}}_{4}}^{2}}{\left|m_{\tilde{g}}\right|^{5}} H\left(\frac{m_{\tilde{\mathcal{D}}_{3}}^{2}}{\left|m_{\tilde{g}}\right|^{2}}, \frac{m_{\tilde{\mathcal{D}}_{4}}^{2}}{\left|m_{\tilde{g}}\right|^{2}}, \frac{m_{b_{i+3}}^{2}}{\left|m_{\tilde{g}}\right|^{2}}\right)\right] .
\end{aligned}
$$

Here, $m_{\tilde{\mathcal{U}}_{i}}(i=1,2,3,4)$ denote the masses of the up type exotic scalar quarks, and $m_{\tilde{\mathcal{D}}_{i}}(i=$ $1,2,3,4)$ are the masses of the down type exotic scalar quarks.

From the couplings of neutral Higgs and exotic quarks in Eq.(41), we obtain their contributions to the Wilson coefficient $C_{5}(\Lambda)$.

$$
\begin{aligned}
C_{5}^{H^{0} t^{\prime} t^{\prime}}= & 2 g_{3}^{3}\left(\frac{1}{4 \pi}\right)^{4} \sum_{\alpha=1}^{8}\left[\sum_{i=1}^{2} \operatorname{Im}\left(\left(\mathcal{N}_{H_{\alpha}^{0}}^{R}\right)_{i i}\left(\mathcal{N}_{H_{\alpha}^{0}}^{L}\right)_{i i}^{\dagger}\right) \frac{1}{m_{t_{i+3}}^{2}} h\left(m_{t_{i+3}}, m_{H_{\alpha}^{0}}\right)\right. \\
& \left.+\operatorname{Im}\left(\left(\mathcal{N}_{H_{\alpha}^{0}}^{R}\right)_{12}\left(\mathcal{N}_{H_{\alpha}^{0}}^{L}\right)_{21}^{\dagger}\right) \frac{1}{m_{t_{4}} m_{t_{5}}} h^{\prime}\left(m_{t_{4}}, m_{t_{5}}, m_{H_{\alpha}^{0}}\right)\right] \\
C_{5}^{H^{0} b^{\prime} b^{\prime}}= & 2 g_{3}^{3}\left(\frac{1}{4 \pi}\right)^{4} \sum_{\alpha=1}^{8}\left[\sum_{i=1}^{2} \operatorname{Im}\left(\left(\mathcal{K}_{H_{\alpha}^{0}}^{R}\right)_{i i}\left(\mathcal{K}_{H_{\alpha}^{0}}^{L}\right)_{i i}^{\dagger}\right) \frac{1}{m_{b_{i+3}}^{2}} h\left(m_{b_{i+3}}, m_{H_{\alpha}^{0}}\right)\right. \\
& \left.+\operatorname{Im}\left(\left(\mathcal{K}_{H_{\alpha}^{0}}^{R}\right)_{12}\left(\mathcal{K}_{H_{\alpha}^{0}}^{L}\right)_{21}^{\dagger}\right) \frac{1}{m_{b_{4}} m_{b_{5}}} h^{\prime}\left(m_{b_{4}}, m_{b_{5}}, m_{H_{\alpha}^{0}}\right)\right]
\end{aligned}
$$

where the functions $h(x, y)$ and $h^{\prime}(x, y, z)$ are given in [21]. In the same way, the contributions from charged Higgs and exotic quarks are written in the following form.

$$
C_{5}^{H^{+} t^{\prime} b^{\prime}}=2 g_{3}^{3}\left(\frac{1}{4 \pi}\right)^{4} \sum_{i, j=1}^{2} \frac{1}{m_{t_{i+3}} m_{b_{j+3}}}\left[\operatorname{Im}\left(\left(\mathcal{N}_{H^{+}}^{R}\right)_{i j}\left(\mathcal{N}_{H^{+}}^{L}\right)_{j i}^{\dagger}\right) h^{\prime}\left(m_{t_{i+3}}, m_{b_{j+3}}, m_{H^{+}}\right)\right.
$$




$$
\left.+\operatorname{Im}\left(\left(\mathcal{N}_{G^{+}}^{R}\right)_{i j}\left(\mathcal{N}_{G^{+}}^{L}\right)_{j i}^{\dagger}\right) h^{\prime}\left(m_{t_{i+3}}, m_{b_{j+3}}, m_{G^{+}}\right)\right]
$$

The coupling of quark-exotic quark-superfield X in Eq.(13) can not only give the corrections to the neutron EDM at one loop level, but also contribute to the Wilson coefficient $C_{5}(\Lambda)$ at two loop level.

$$
\begin{aligned}
C_{5}^{X q^{\prime} q}=2 & g_{3}^{3}\left(\frac{1}{4 \pi}\right)^{4} \sum_{i, j=1}^{2}\left[\operatorname{Im}\left(\left(\mathcal{N}_{t^{\prime}}^{R}\right)_{i j}\left(\mathcal{N}_{t^{\prime}}^{L}\right)_{j i}^{\dagger}\right) \frac{1}{m_{t_{i+3}} m_{t}} h^{\prime}\left(m_{t_{i+3}}, m_{t}, m_{X_{j}}\right)\right. \\
& \left.+\operatorname{Im}\left(\left(\mathcal{N}_{b^{\prime}}^{R}\right)_{i j}\left(\mathcal{N}_{b^{\prime}}^{L}\right)_{j i}^{\dagger}\right) \frac{1}{m_{b_{i+3}} m_{b}} h^{\prime}\left(m_{b_{i+3}}, m_{b}, m_{X_{j}}\right)\right] .
\end{aligned}
$$

The results obtained at the matching scale $\Lambda$ should be transformed down to the chirality breaking scale $\Lambda_{\chi}$ [22]. So the renormalization group equations (RGEs) for the Wilson coefficients of the Weinberg operator and the quark EDMs, CEDMs should be solved. The relations between the results at two deferent scales $\Lambda$ and $\Lambda_{\chi}$ are presented here.

$$
d_{q}^{\gamma}\left(\Lambda_{\chi}\right)=1.53 d_{q}^{\gamma}(\Lambda), \quad d_{q}^{g}\left(\Lambda_{\chi}\right)=3.4 d_{q}^{g}(\Lambda), \quad C_{5}\left(\Lambda_{\chi}\right)=3.4 C_{5}(\Lambda)
$$

There are three type contributions to the quark EDM, 1 the electroweak contribution $d_{q}^{\gamma}, 2$ the CDEM contribution $d_{q}^{g}$, 3 the Weinberg operator contribution. Each type contribution can be calculated numerically. At a low scale, the quark EDM can be obtained from $d_{q}^{\gamma}, d_{q}^{g}$ and $C_{5}\left(\Lambda_{\chi}\right)$ by the following formula[23].

$$
d_{q}=d_{q}^{\gamma}+\frac{e}{4 \pi} d_{q}^{g}+\frac{e \Lambda_{\chi}}{4 \pi} C_{5}\left(\Lambda_{\chi}\right)
$$

From the quark model, the EDM of neutron is derived from $\mathrm{u}$ quark's EDM $d_{u}$ and $\mathrm{d}$ quark's EDM $d_{d}$ with the following expression

$$
d_{n}=\frac{1}{3}\left(4 d_{d}-d_{u}\right)
$$

\section{THE NUMERICAL ANALYSIS}

The lightest neutral CP even Higgs is discovered by LHC, with a mass $m_{h_{0}} \simeq 125.9 \mathrm{GeV}$, which gives the most stringent constraint on the parameter space. Furthermore, the ATLAS and CMS experiments announce the Higgs production and decay into diphoton channel are 
larger than SM predictions with a factor $1.2 \sim 2$. The current values of the ratios are [9, 24]: $R_{\gamma \gamma}=1.6 \pm 0.4, R_{V V^{*}}=0.94 \pm 0.40,(V=Z, W)$. CMS collaboration declare that it is impossible to find a SM Higgs with mass from 127.5 to $600 \mathrm{GeV}$. Considering all the above constraints, we obtain a parameter space in our recent work [10].

In this work to obtain the numerical results, the relevant parameters in SM are shown here.

$$
\begin{aligned}
& \alpha_{s}\left(m_{\mathrm{z}}\right)=0.118, \quad \alpha\left(m_{\mathrm{z}}\right)=1 / 128, \quad s_{\mathrm{w}}^{2}\left(m_{\mathrm{z}}\right)=0.23, \\
& m_{\mathrm{w}}=80.4 \mathrm{GeV}, m_{t}=174.2 \mathrm{GeV}, \quad m_{b}=4.2 \mathrm{GeV}, \\
& m_{u}=2.3 \times 10^{-3} \mathrm{GeV}, \quad m_{d}=4.8 \times 10^{-3} \mathrm{GeV},
\end{aligned}
$$

To simplify the numerical discussion, we suppose $A_{t}, A_{b}, A_{\nu_{4}}, A_{e_{4}}, A_{\nu_{5}}, A_{\nu_{5}}, A_{u_{4}}, A_{u_{5}}, A_{d_{4}}, A_{d_{5}}$, $A_{B Q}, A_{B U}, A_{B D}$ and $Y_{u_{4}}, Y_{u_{5}}, Y_{d_{4}}, Y_{d_{5}}$ are all real parameters.

The other parameters are adopted[10].

$$
\begin{aligned}
& \tan \beta=\tan \beta_{B}=\tan \beta_{L}=2, \quad B_{4}=L_{4}=\frac{3}{2} \\
& m_{\tilde{Q}_{3}}=m_{\tilde{U}_{3}}=m_{\tilde{D}_{3}}=1 \mathrm{TeV}, \quad m_{Z_{B}}=m_{Z_{L}}=1 \mathrm{TeV} \\
& m_{\tilde{U}_{4}}=m_{\tilde{D}_{4}}=m_{\tilde{Q}_{5}}=m_{\tilde{U}_{5}}=m_{\tilde{D}_{5}}=1 \mathrm{TeV}, m_{\tilde{Q}_{4}}=790 \mathrm{GeV}, \\
& m_{\tilde{L}_{4}}=m_{\tilde{\nu}_{4}}=m_{\tilde{E}_{4}}=m_{\tilde{L}_{5}}=m_{\tilde{\nu}_{5}}=m_{\tilde{E}_{5}}=1 \mathrm{TeV}, \\
& A_{\nu_{4}}=A_{e_{4}}=A_{\nu_{5}}=A_{\nu_{5}}=A_{u_{4}}=A_{u_{5}}=A_{d_{4}}=A_{d_{5}}=550 \mathrm{GeV}, \\
& v_{B_{t}}=\sqrt{v_{B}^{2}+\bar{v}_{B}^{2}}=3 \mathrm{TeV}, \quad v_{L_{t}}=\sqrt{v_{L}^{2}+\bar{v}_{L}^{2}}=3 \mathrm{TeV} \\
& A_{B Q}=A_{B U}=A_{B D}=-A_{b}=-A_{t}=1 \mathrm{TeV}, \quad m_{1}=\mathrm{TeV} \\
& Y_{u_{4}}=0.76 Y_{t}, Y_{d_{4}}=0.7 Y_{b}, Y_{u_{5}}=0.7 Y_{b}, Y_{d_{5}}=0.13 Y_{t}, \\
& m_{2}=750 \mathrm{GeV}, \mu_{B}=500 \mathrm{GeV}, \Lambda=1 \mathrm{TeV}, \Lambda_{\chi}=1 \mathrm{GeV}, \\
& \lambda_{Q}=\lambda_{u}=\lambda_{d}=0.5, \quad\left|m_{\tilde{g}}\right|=300 \mathrm{GeV}, \quad B_{B}=1 \mathrm{TeV}, \\
& m_{\nu_{4}}=m_{\nu_{5}}=90 \mathrm{GeV}, \quad m_{e_{4}}=m_{e_{5}}=100 \mathrm{GeV}, \quad m_{\mu}=-800 \mathrm{GeV} .
\end{aligned}
$$

The CP violating phase $\theta_{3}$ of gluino affects the neutron EDM obviously. Supposing $B_{X}=300 \mathrm{GeV}, \lambda_{1}=\lambda_{2}=\lambda_{3}=0.1,\left|\mu_{X}\right|=2400 \mathrm{GeV}$ and the $\mathrm{CP}$ violating phase of $\mu_{X}$ is zero $\left(\theta_{X}=0\right)$, we plot the neutron EDM $d_{n}$ varying with the phase $\theta_{3}$ in Fig.(15), with the 
condition $m_{\tilde{Q}_{1}}=m_{\tilde{U}_{1}}=m_{\tilde{D}_{1}}=M Q$. When $M Q=1000 \mathrm{GeV}$ and $\theta_{3}= \pm \frac{\pi}{2}$, the absolute value of the neutron $\mathrm{EDM} d_{n}$ reaches the biggest value $1.04 \times 10^{-25}(e . \mathrm{cm})$, which is very close to the present experiment upper limit. For the same value of $\theta_{3}$, the absolute value of $d_{n}$ with $M Q=1200 \mathrm{GeV}$ is smaller than that with $M Q=1000 \mathrm{GeV}$.

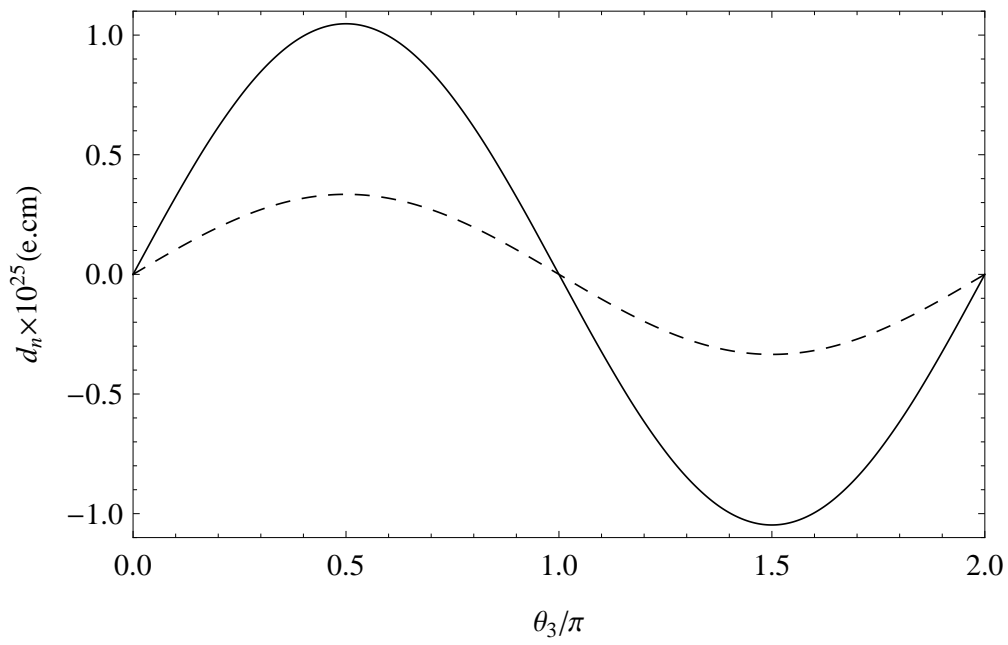

FIG. 5: As $B_{X}=300 \mathrm{GeV}, \lambda_{1}=\lambda_{2}=\lambda_{3}=0.1,\left|\mu_{X}\right|=2400 \mathrm{GeV}, \theta_{X}=0$ and $m_{\tilde{Q}_{1}}=m_{\tilde{U}_{1}}=$ $m_{\tilde{D}_{1}}=M Q$, the solid-line and dashed-line represent $d_{n}$ varying with $\theta_{3}$, for $M Q=1000 \mathrm{GeV}$ and $1200 \mathrm{GeV}$ respectively.

Then one finds $M Q$ affects the theoretical results strongly. Taking $B_{X}=300 \mathrm{GeV}, \lambda_{1}=$ $\lambda_{2}=\lambda_{3}=0.1,\left|\mu_{X}\right|=2400 \mathrm{GeV}, \theta_{X}=0, \theta_{3}=\frac{\pi}{2}\left(\frac{\pi}{4}\right)$, and $m_{\tilde{Q}_{1}}=m_{\tilde{U}_{1}}=m_{\tilde{D}_{1}}=M Q$, the neutron EDM $d_{n}$ varying with $M Q$ is plotted in Fig.(6). Obviously, $d_{n}$ becomes small quickly, with the enlarging $M Q$ in the region from $1000 \mathrm{GeV}$ to $1500 \mathrm{GeV}$. When $M Q=2000 \mathrm{GeV}$, $d_{n}$ turns very small, whose value is around $5 \times 10^{-28}($ e.cm) .

The superfields X can give contributions to the neutron EDM $d_{n}$ at one-loop level, so the parameters having relation with the mass squared matrix of superfields $\mathrm{X}$ and the couplings of superfields X-exotic quark-quark can affect the theoretical predictions strongly. With the assumption in the parameter space $B_{X}=300 \mathrm{GeV}, \lambda_{1}=\lambda_{2}=\lambda_{3}=0.1, \theta_{3}=0$ and $m_{\tilde{Q}_{1}}=m_{\tilde{U}_{1}}=m_{\tilde{D}_{1}}=1000 \mathrm{GeV}$, we investigate the relation between $d_{n}$ and the $\mathrm{CP}$ violating phase $\theta_{X}$ numerically in Fig.(17). The shapes of the diagrams in Fig.(7) are very similar as those in Fig.(15). The biggest absolute value of $d_{n}$ is obtained with $\theta_{X}=\frac{\pi}{2}$ and 


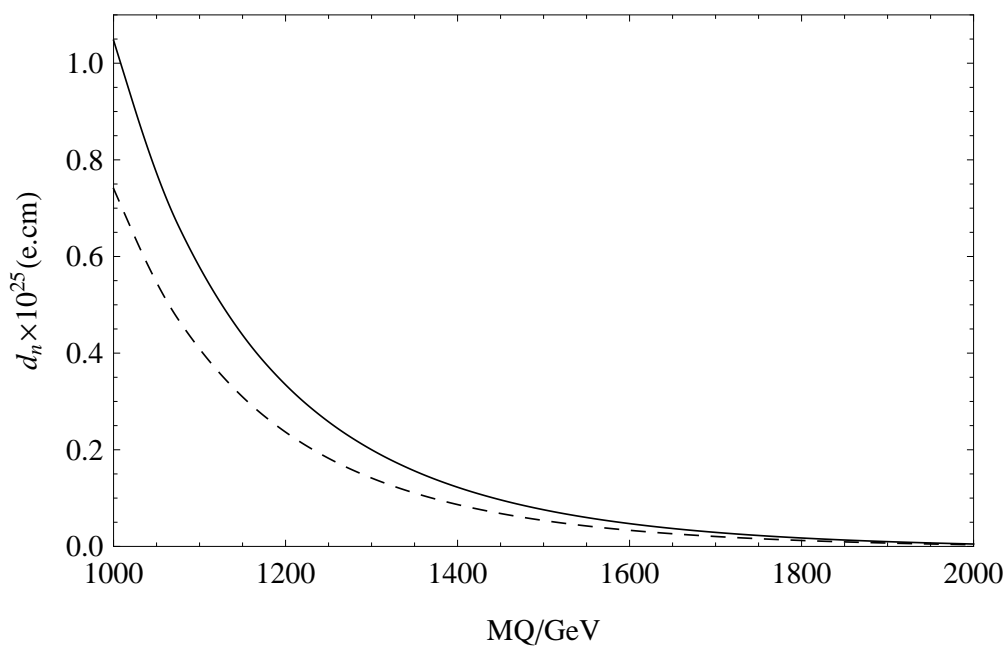

FIG. 6: As $B_{X}=300 \mathrm{GeV}, \lambda_{1}=\lambda_{2}=\lambda_{3}=0.1,\left|\mu_{X}\right|=2400 \mathrm{GeV}, \theta_{X}=0$ and $m_{\tilde{Q}_{1}}=m_{\tilde{U}_{1}}=$ $m_{\tilde{D}_{1}}=M Q$, the solid-line and dashed-line represent $d_{n}$ varying with $M Q$, for $\theta_{3}=\frac{\pi}{2}$ and $\frac{\pi}{4}$ respectively.

$\left|\mu_{X}\right|=2400 \mathrm{GeV}$. When $\left|\mu_{X}\right|=3000 \mathrm{GeV}$, the absolute value of $d_{n}$ is smaller than that with $\left|\mu_{X}\right|=2400 \mathrm{GeV}$.

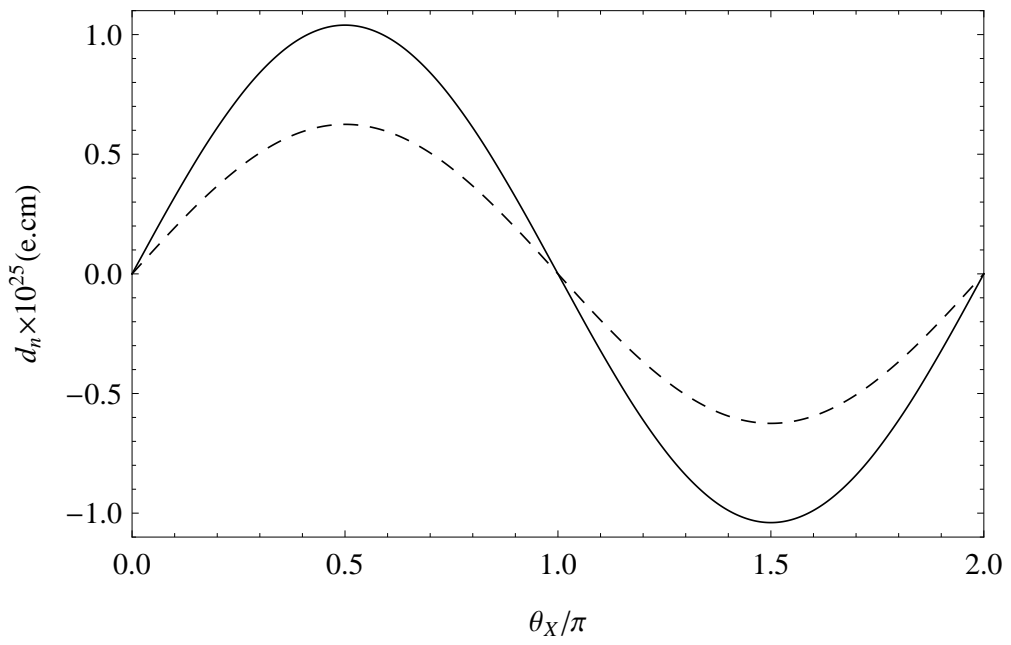

FIG. 7: As $B_{X}=300 \mathrm{GeV}, \lambda_{1}=\lambda_{2}=\lambda_{3}=0.1, \theta_{3}=0$ and $m_{\tilde{Q}_{1}}=m_{\tilde{U}_{1}}=m_{\tilde{D}_{1}}=1000 \mathrm{GeV}$, the solid-line and dashed-line represent $d_{n}$ varying with $\theta_{X}$, for $\left|\mu_{X}\right|=2400 \mathrm{GeV}$ and $\left|\mu_{X}\right|=3000 \mathrm{GeV}$ respectively.

In Fig.(8), we can easily find the neutron EDM turns smaller and smaller, as the absolute 
value of $\mu_{x}$ becomes larger and larger. The reason should be that some imaginary parts come from the non-diagonal elements in the diagonalizing matrix $Z_{X}$. Large diagonalized elements in the mass squared matrix of the superfields X lead to small non-diagonal elements in $Z_{X}$. Therefore, we obtain the small theoretical prediction on the neutron EDM.

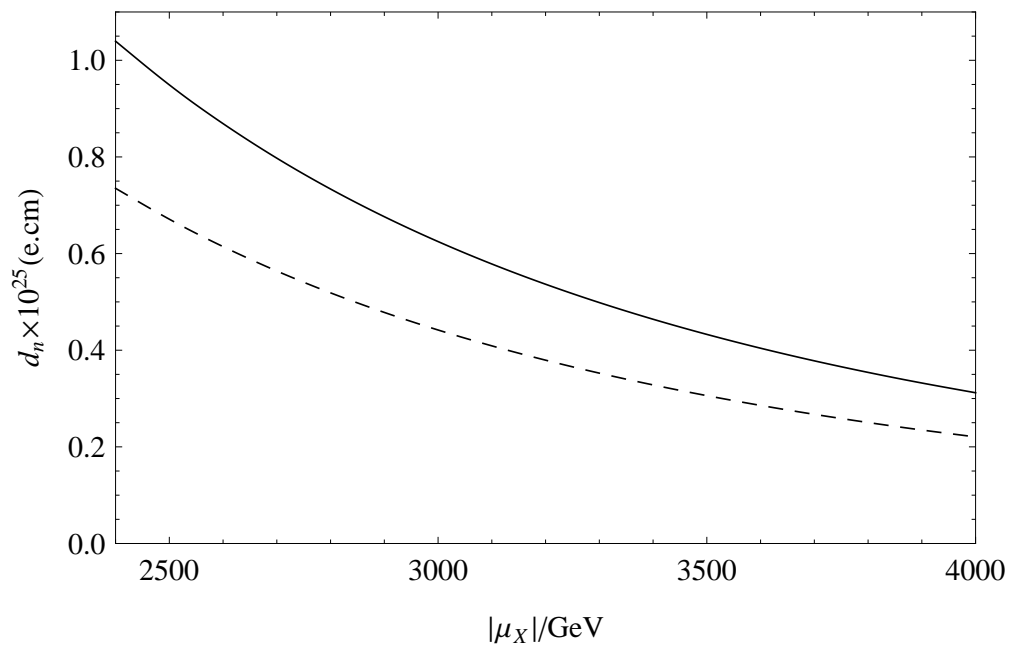

FIG. 8: As $B_{X}=300 \mathrm{GeV}, \lambda_{1}=\lambda_{2}=\lambda_{3}=0.1, \theta_{3}=0$ and $m_{\tilde{Q}_{1}}=m_{\tilde{U}_{1}}=m_{\tilde{D}_{1}}=1000 \mathrm{GeV}$, the solid-line and dashed-line represent $d_{n}$ varying with $\left|\mu_{X}\right|$, for $\theta_{X}=\frac{\pi}{2}$ and $\frac{\pi}{4}$ respectively.

Choosing $\lambda_{1}=\lambda_{2}=\lambda_{3}=0.1, \theta_{3}=0, \theta_{X}=\frac{\pi}{2}, m_{\tilde{Q}_{1}}=m_{\tilde{U}_{1}}=m_{\tilde{D}_{1}}=1000 \mathrm{GeV}$, we plot $d_{n}$ varying with the parameter $B_{X}$ with $\left|\mu_{X}\right|=2400$ (3000) GeV in Fig.(9). From Fig.(9), the neutron EDM $d_{n}$ is almost the linear increasing function of $B_{X}$. Because $B_{X}$ is the non-diagonal element in the mass squared matrix of the superfields $\mathrm{X}$, large $B_{X}$ gives big contribution to imaginary elements in the matrix $Z_{X}$.

From the Lagrangian of quark-exotic quark and the superfields X in Eqs.(13) and (14), one finds $\lambda_{1}, \lambda_{2}, \lambda_{3}$ are important parameters, and they affect those coupling strongly. So, we analyse how $\lambda_{1}, \lambda_{2}, \lambda_{3}$ influence the neutron EDM with the hypothesis in parameter space as $B_{X}=300 \mathrm{GeV}, \theta_{3}=0, \theta_{X}=\frac{\pi}{2}, m_{\tilde{Q}_{1}}=m_{\tilde{U}_{1}}=m_{\tilde{D}_{1}}=1000 \mathrm{GeV}$ and $\lambda_{1}=\lambda_{2}=\lambda_{3}=$ Lam. The numerical results are plotted in the Fig.(10) for $\mu_{X}=2400$ (3000) GeV. When Lam is very small, neutron EDM $d_{n}$ is almost zero. The diagrams in Fig.(10) is symmetrical for the axis $\operatorname{Lam}=0$. 


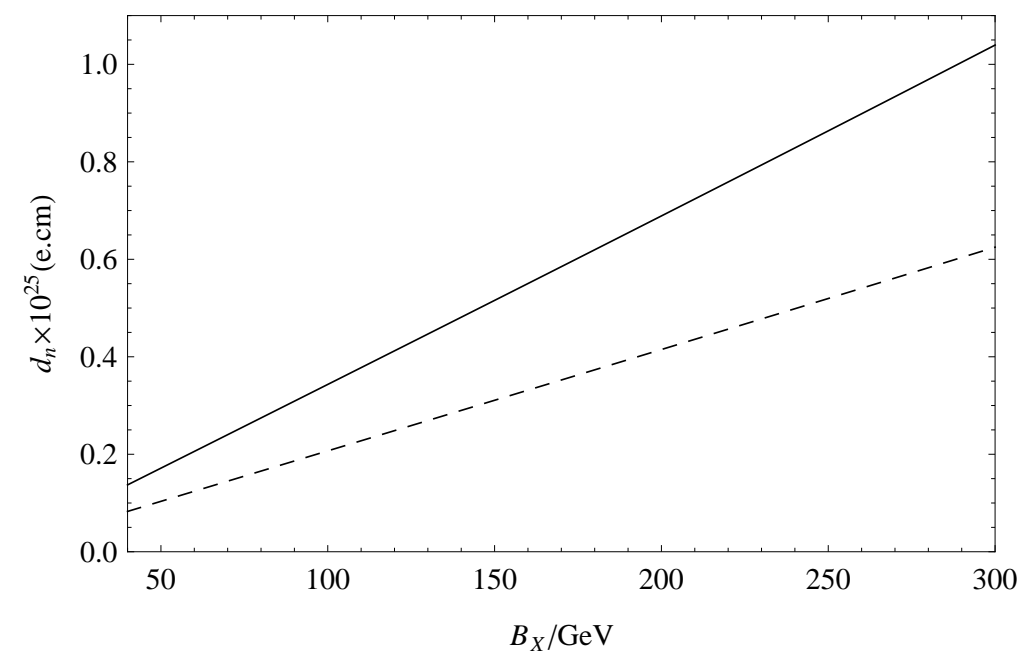

FIG. 9: As $\lambda_{1}=\lambda_{2}=\lambda_{3}=0.1, \theta_{3}=0, \theta_{X}=\frac{\pi}{2}$ and $m_{\tilde{Q}_{1}}=m_{\tilde{U}_{1}}=m_{\tilde{D}_{1}}=1000 \mathrm{GeV}$, the solid-line and dashed-line represent $d_{n}$ varying with $B_{X}$, for $\left|\mu_{X}\right|=2400 \mathrm{GeV}$ and $3000 \mathrm{GeV}$ respectively.

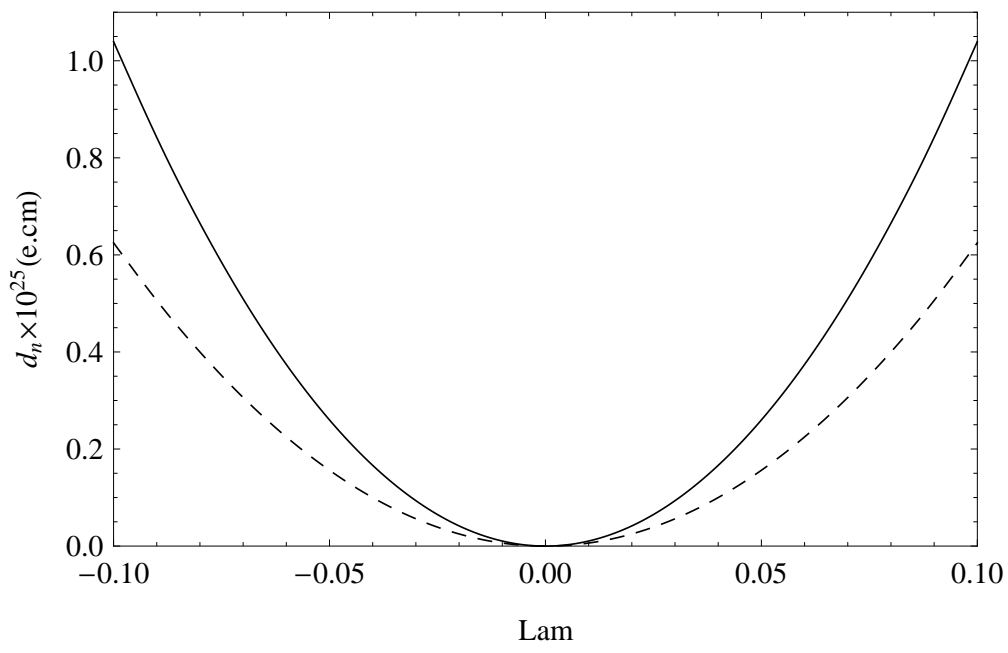

FIG. 10: As $B_{X}=300 \mathrm{GeV}, \theta_{3}=0, \theta_{X}=\frac{\pi}{2}, m_{\tilde{Q}_{1}}=m_{\tilde{U}_{1}}=m_{\tilde{D}_{1}}=1000 \mathrm{GeV}$ and $\lambda_{1}=\lambda_{2}=$ $\lambda_{3}=L a m$, the solid-line and dashed-line represent $d_{n}$ varying with $L a m$, for $\left|\mu_{X}\right|=2400 \mathrm{GeV}$ and $3000 \mathrm{GeV}$ respectively.

\section{SUMMARY}

We study the neutron EDM in the framework of the BLMSSM. The constraints from the experiment data on the lightest CP-even Higgs mass $\left(m_{h^{0}}=125.9 \mathrm{GeV}\right)$ and Higgs decay 
into diphoton $\left(Z Z^{*}, W W^{*}\right)$ are all considered. Furthermore the confine on the heaviest CP-even Higgs and CP-odd Higgs mass is also taking into account $\left(m_{0}, m_{0}\right)>600 \mathrm{GeV}$. We analyse how the neutron EDM depends on the related parameters. If the new physics mass at $\mathrm{TeV}$ range, the present experiment upper bound on the neutron EDM gives a serious constraint on the $\mathrm{CP}$ violating phase and the BLMSSM parameters. Our numerical results show that in some parameter space, the neutron EDM $d_{n}$ can reach it's present experimental upper bound $1.1 \times 10^{-25}($ e.cm $)$.

\section{Appendix A: The couplings between Higgs and exotic quarks}

The coupling constants $\left(\mathcal{N}_{H_{\alpha}^{0}}^{L}\right)(\alpha=1,2 \cdots 8)$ for neutral Higgs and up type exotic quarks are expressed as

$$
\begin{aligned}
\left(\mathcal{N}_{H_{1}^{0}}^{L}\right)_{i j}= & \frac{1}{\sqrt{2}}\left[Y_{u_{4}}\left(W_{t}^{\dagger}\right)_{i 2}\left(U_{t}\right)_{1 j} \cos \alpha+Y_{u_{5}}\left(W_{t}^{\dagger}\right)_{i 1}\left(U_{t}\right)_{2 j} \sin \alpha\right], \\
\left(\mathcal{N}_{H_{1}^{0}}^{R}\right)_{i j}= & \frac{1}{\sqrt{2}}\left[Y_{u_{4}}^{*}\left(U_{t}^{\dagger}\right)_{i 1}\left(W_{t}\right)_{2 j} \cos \alpha+Y_{u_{5}}^{*}\left(U_{t}^{\dagger}\right)_{i 2}\left(W_{t}\right)_{1 j} \sin \alpha\right], \\
\left(\mathcal{N}_{H_{2}^{0}}^{L}\right)_{i j}= & \frac{1}{\sqrt{2}}\left[Y_{u_{4}}\left(W_{t}^{\dagger}\right)_{i 2}\left(U_{t}\right)_{1 j} \sin \alpha-Y_{u_{5}}\left(W_{t}^{\dagger}\right)_{i 1}\left(U_{t}\right)_{2 j} \cos \alpha\right], \\
\left(\mathcal{N}_{H_{2}^{0}}^{R}\right)_{i j}= & \frac{1}{\sqrt{2}}\left[Y_{u_{4}}^{*}\left(U_{t}^{\dagger}\right)_{i 1}\left(W_{t}\right)_{2 j} \sin \alpha-Y_{u_{5}}^{*}\left(U_{t}^{\dagger}\right)_{i 2}\left(W_{t}\right)_{1 j} \cos \alpha\right], \\
\left(\mathcal{N}_{H_{3}^{0}}^{L}\right)_{i j}= & \frac{i}{\sqrt{2}}\left[Y_{u_{4}}\left(W_{t}^{\dagger}\right)_{i 2}\left(U_{t}\right)_{1 j} \cos \beta+Y_{u_{5}}\left(W_{t}^{\dagger}\right)_{i 1}\left(U_{t}\right)_{2 j} \sin \beta\right], \\
\left(\mathcal{N}_{H_{3}^{0}}^{R}\right)_{i j}= & -\frac{i}{\sqrt{2}}\left[Y_{u_{4}}^{*}\left(U_{t}^{\dagger}\right)_{i 1}\left(W_{t}\right)_{2 j} \cos \beta+Y_{u_{5}}^{*}\left(U_{t}^{\dagger}\right)_{i 2}\left(W_{t}\right)_{1 j} \sin \beta\right], \\
\left(\mathcal{N}_{H_{4}^{0}}^{L}\right)_{i j}= & \frac{i}{\sqrt{2}}\left[Y_{u_{4}}\left(W_{t}^{\dagger}\right)_{i 2}\left(U_{t}\right)_{1 j} \sin \beta-Y_{u_{5}}\left(W_{t}^{\dagger}\right)_{i 1}\left(U_{t}\right)_{2 j} \cos \beta\right], \\
\left(\mathcal{N}_{H_{4}^{0}}^{R}\right)_{i j}= & -\frac{i}{\sqrt{2}}\left[Y_{u_{4}}^{*}\left(U_{t}^{\dagger}\right)_{i 1}\left(W_{t}\right)_{2 j} \sin \beta-Y_{u_{5}}^{*}\left(U_{t}^{\dagger}\right)_{i 2}\left(W_{t}\right)_{1 j} \cos \beta\right], \\
\left(\mathcal{N}_{H_{5}^{0}}^{L}\right)_{i j}= & -\frac{1}{\sqrt{2}}\left[\lambda_{u}\left(W_{t}^{\dagger}\right)_{i 2}\left(U_{t}\right)_{2 j} \cos \alpha_{B}-\lambda_{Q}\left(W_{t}^{\dagger}\right)_{i 1}\left(U_{t}\right)_{1 j} \sin \alpha_{B}\right], \\
\left(\mathcal{N}_{H_{5}^{0}}^{R}\right)_{i j}= & -\frac{1}{\sqrt{2}}\left[\lambda_{u}^{*}\left(U_{t}^{\dagger}\right)_{i 2}\left(W_{t}\right)_{2 j} \cos \alpha_{B}-\lambda_{Q}^{*}\left(U_{t}^{\dagger}\right)_{i 2}\left(W_{t}\right)_{2 j} \sin \alpha_{B}\right], \\
\left(\mathcal{N}_{H_{6}^{0}}^{L}\right)_{i j}= & -\frac{1}{\sqrt{2}}\left[\lambda_{u}\left(W_{t}^{\dagger}\right)_{i 2}\left(U_{t}\right)_{2 j} \sin \alpha_{B}+\lambda_{Q}\left(W_{t}^{\dagger}\right)_{i 1}\left(U_{t}\right)_{1 j} \cos \alpha_{B}\right], \\
\left(\mathcal{N}_{H_{6}^{0}}^{R}\right)_{i j}= & -\frac{1}{\sqrt{2}}\left[\lambda_{u}^{*}\left(U_{t}^{\dagger}\right)_{i 2}\left(W_{t}\right)_{2 j} \sin \alpha_{B}+\lambda_{Q}^{*}\left(U_{t}^{\dagger}\right)_{i 2}\left(W_{t}\right)_{2 j} \cos \alpha_{B}\right], \\
\left(\mathcal{N}_{H_{7}^{0}}^{L}\right)_{i j}= & -\frac{i}{\sqrt{2}}\left[\lambda_{u}\left(W_{t}^{\dagger}\right)_{i 2}\left(U_{t}\right)_{2 j} \cos \beta_{B}-\lambda_{Q}\left(W_{t}^{\dagger}\right)_{i 1}\left(U_{t}\right)_{1 j} \sin \beta_{B}\right],
\end{aligned}
$$




$$
\begin{aligned}
\left(\mathcal{N}_{H_{7}^{0}}^{R}\right)_{i j} & =\frac{i}{\sqrt{2}}\left[\lambda_{u}^{*}\left(U_{t}^{\dagger}\right)_{i 2}\left(W_{t}\right)_{2 j} \cos \beta_{B}-\lambda_{Q}^{*}\left(U_{t}^{\dagger}\right)_{i 2}\left(W_{t}\right)_{2 j} \sin \beta_{B}\right], \\
\left(\mathcal{N}_{H_{8}^{0}}^{L}\right)_{i j} & =-\frac{i}{\sqrt{2}}\left[\lambda_{u}\left(W_{t}^{\dagger}\right)_{i 2}\left(U_{t}\right)_{2 j} \sin \beta_{B}+\lambda_{Q}\left(W_{t}^{\dagger}\right)_{i 1}\left(U_{t}\right)_{1 j} \cos \beta_{B}\right], \\
\left(\mathcal{N}_{H_{8}^{0}}^{R}\right)_{i j} & =\frac{i}{\sqrt{2}}\left[\lambda_{u}^{*}\left(U_{t}^{\dagger}\right)_{i 2}\left(W_{t}\right)_{2 j} \sin \beta_{B}+\lambda_{Q}^{*}\left(U_{t}^{\dagger}\right)_{i 2}\left(W_{t}\right)_{2 j} \cos \beta_{B}\right] .
\end{aligned}
$$

We show the coupling constants $\left(\mathcal{N}_{H_{\alpha}^{0}}^{L}\right)(\alpha=1,2 \cdots 8)$ for neutral Higgs and down type exotic quarks here.

$$
\begin{aligned}
& \left(\mathcal{K}_{H_{1}^{0}}^{L}\right)_{i j}=\frac{1}{\sqrt{2}}\left[Y_{d_{4}}\left(W_{b}^{\dagger}\right)_{i 2}\left(U_{b}\right)_{1 j} \sin \alpha-Y_{d_{5}}\left(W_{b}^{\dagger}\right)_{i 1}\left(U_{b}\right)_{2 j} \cos \alpha\right], \\
& \left(\mathcal{K}_{H_{1}^{0}}^{R}\right)_{i j}=\frac{1}{\sqrt{2}}\left[Y_{d_{4}}^{*}\left(U_{b}^{\dagger}\right)_{i 1}\left(W_{b}\right)_{2 j} \sin \alpha-Y_{d_{5}}^{*}\left(U_{b}^{\dagger}\right)_{i 2}\left(W_{b}\right)_{1 j} \cos \alpha\right], \\
& \left(\mathcal{K}_{H_{2}^{0}}^{L}\right)_{i j}=-\frac{1}{\sqrt{2}}\left[Y_{d_{4}}\left(W_{b}^{\dagger}\right)_{i 2}\left(U_{b}\right)_{1 j} \cos \alpha+Y_{d_{5}}\left(W_{b}^{\dagger}\right)_{i 1}\left(U_{b}\right)_{2 j} \sin \alpha\right] \text {, } \\
& \left(\mathcal{K}_{H_{2}^{0}}^{R}\right)_{i j}=-\frac{1}{\sqrt{2}}\left[Y_{d_{4}}^{*}\left(U_{b}^{\dagger}\right)_{i 1}\left(W_{b}\right)_{2 j} \cos \alpha+Y_{d_{5}}^{*}\left(U_{b}^{\dagger}\right)_{i 2}\left(W_{b}\right)_{1 j} \sin \alpha\right] \text {, } \\
& \left(\mathcal{K}_{H_{3}^{0}}^{L}\right)_{i j}=\frac{i}{\sqrt{2}}\left[Y_{d_{4}}\left(W_{b}^{\dagger}\right)_{i 2}\left(U_{b}\right)_{1 j} \sin \beta-Y_{d_{5}}\left(W_{b}^{\dagger}\right)_{i 1}\left(U_{b}\right)_{2 j} \cos \beta\right] \text {, } \\
& \left(\mathcal{K}_{H_{3}^{0}}^{R}\right)_{i j}=-\frac{i}{\sqrt{2}}\left[Y_{d_{4}}^{*}\left(U_{b}^{\dagger}\right)_{i 1}\left(W_{b}\right)_{2 j} \sin \beta-Y_{d_{5}}^{*}\left(U_{b}^{\dagger}\right)_{i 2}\left(W_{b}\right)_{1 j} \cos \beta\right], \\
& \left(\mathcal{K}_{H_{4}^{0}}^{L}\right)_{i j}=-\frac{i}{\sqrt{2}}\left[Y_{d_{4}}\left(W_{b}^{\dagger}\right)_{i 2}\left(U_{b}\right)_{1 j} \cos \beta+Y_{d_{5}}\left(W_{b}^{\dagger}\right)_{i 1}\left(U_{b}\right)_{2 j} \sin \beta\right] \text {, } \\
& \left(\mathcal{K}_{H_{4}^{0}}^{R}\right)_{i j}=\frac{i}{\sqrt{2}}\left[Y_{d_{4}}^{*}\left(U_{b}^{\dagger}\right)_{i 1}\left(W_{b}\right)_{2 j} \cos \beta+Y_{d_{5}}^{*}\left(U_{b}^{\dagger}\right)_{i 2}\left(W_{b}\right)_{1 j} \sin \beta\right], \\
& \left(\mathcal{K}_{H_{5}^{0}}^{L}\right)_{i j}=-\frac{1}{\sqrt{2}}\left[\lambda_{d}\left(W_{b}^{\dagger}\right)_{i 2}\left(U_{b}\right)_{2 j} \cos \alpha_{B}+\lambda_{Q}\left(W_{b}^{\dagger}\right)_{i 1}\left(U_{b}\right)_{1 j} \sin \alpha_{B}\right] \text {, } \\
& \left(\mathcal{K}_{H_{5}^{0}}^{R}\right)_{i j}=-\frac{1}{\sqrt{2}}\left[\lambda_{d}^{*}\left(U_{b}^{\dagger}\right)_{i 2}\left(W_{b}\right)_{2 j} \cos \alpha_{B}+\lambda_{Q}^{*}\left(U_{b}^{\dagger}\right)_{i 2}\left(W_{b}\right)_{2 j} \sin \alpha_{B}\right] \text {, } \\
& \left(\mathcal{K}_{H_{6}^{0}}^{L}\right)_{i j}=-\frac{1}{\sqrt{2}}\left[\lambda_{d}\left(W_{b}^{\dagger}\right)_{i 2}\left(U_{b}\right)_{2 j} \sin \alpha_{B}+\lambda_{Q}\left(W_{b}^{\dagger}\right)_{i 1}\left(U_{b}\right)_{1 j} \cos \alpha_{B}\right], \\
& \left(\mathcal{K}_{H_{6}^{0}}^{R}\right)_{i j}=-\frac{1}{\sqrt{2}}\left[\lambda_{d}^{*}\left(U_{b}^{\dagger}\right)_{i 2}\left(W_{b}\right)_{2 j} \sin \alpha_{B}+\lambda_{Q}^{*}\left(U_{b}^{\dagger}\right)_{i 2}\left(W_{b}\right)_{2 j} \cos \alpha_{B}\right] \text {, } \\
& \left(\mathcal{K}_{H_{7}^{0}}^{L}\right)_{i j}=-\frac{i}{\sqrt{2}}\left[\lambda_{d}\left(W_{b}^{\dagger}\right)_{i 2}\left(U_{b}\right)_{2 j} \cos \beta_{B}+\lambda_{Q}\left(W_{b}^{\dagger}\right)_{i 1}\left(U_{b}\right)_{1 j} \sin \beta_{B}\right] \text {, } \\
& \left(\mathcal{K}_{H_{7}^{0}}^{R}\right)_{i j}=\frac{i}{\sqrt{2}}\left[\lambda_{d}^{*}\left(U_{b}^{\dagger}\right)_{i 2}\left(W_{b}\right)_{2 j} \cos \beta_{B}+\lambda_{Q}^{*}\left(U_{b}^{\dagger}\right)_{i 2}\left(W_{b}\right)_{2 j} \sin \beta_{B}\right] \text {, } \\
& \left(\mathcal{K}_{H_{8}^{0}}^{L}\right)_{i j}=-\frac{i}{\sqrt{2}}\left[\lambda_{d}\left(W_{b}^{\dagger}\right)_{i 2}\left(U_{b}\right)_{2 j} \sin \beta_{B}+\lambda_{Q}\left(W_{b}^{\dagger}\right)_{i 1}\left(U_{b}\right)_{1 j} \cos \beta_{B}\right] \text {, } \\
& \left(\mathcal{K}_{H_{8}^{0}}^{R}\right)_{i j}=\frac{i}{\sqrt{2}}\left[\lambda_{d}^{*}\left(U_{b}^{\dagger}\right)_{i 2}\left(W_{b}\right)_{2 j} \sin \beta_{B}+\lambda_{Q}^{*}\left(U_{b}^{\dagger}\right)_{i 2}\left(W_{b}\right)_{2 j} \cos \beta_{B}\right] \text {. }
\end{aligned}
$$




\section{Acknowledgments}

The work has been supported by the National Natural Science Foundation of China (NNSFC) with Grant No. 11275036, No. 11047002, the Fund of Natural Science Foundation of Hebei Province(A2011201118), the open project of State Key Laboratory of Chinese Academy of Sciences Institute of theoretical physics(Y3KF311CJ1) and Natural Science Fund of Hebei University with Grant No. 2011JQ05, No. 2012-242.

[1] J. Rosiek, Phys.Rev. D 41 (1990) 3464; arXiv:hep-ph/9511250; Tai-Fu Feng, Xiu-Yi Yang, Nucl.Phys. B 814 (2009) 101; H.P. Nilles, Phys. Rept. 110 (1984) 1; H.E. Haber and G.L. Kane, Phys. Rept. 117 (1985); Hai-Bin Zhang, Tai-Fu Feng, Zhao-Feng Ge, Guo-Hui Luo, Shu-Min Zhao, arXiv:hep-ph/1305.4352.

[2] P. F. Perez, Phys. Lett. B 711 (2012) 353; J. M. Arnold, P. F. Perez, B. Fornal, and S. Spinner, Phys. Rev. D 85 (2012)115024.

[3] P. F. Perez and M. B. Wise, JHEP 1108 (2011) 068; Phys. Rev. D 82 (2010) 011901;

[4] Supersymmetry Searches at CMS, http://cms.web.cern.ch/org/cms-papers-and-results.

[5] Supersymmetry Searches at ATLAS, https://twiki.cern.ch/twiki/bin/view/ AtlasPublic/SupersymmetryPublicResults.

[6] P.F. Perez, S. Spinner, arXiv:1308.0524 [hep-ph].

[7] P.F. Perez , M.B. Wise. Phys. Rev. D 84 (2011)055015.

[8] J.M. Butterworth, J.R. Ellis, A.R. Raklev, G.P. Salam, Phys. Rev. Lett. 103 (2009)241803.

[9] CMS Collaboration, Phys. Lett. B 716 (2012) 30; ATLAS Collaboration, Phys. Lett. B 716 (2012) 1; CMS Collaboration arXiv:hep-ph/1301.3405.

[10] Tai-Fu Feng, Shu-Min Zhao, Hai-Bin Zhang, Yin-Jie Zhang, Yu-Li Yan, Nucl.Phys. B 871 (2013) 223.

[11] S. Heinemeyer, D. Stockinger and G. Weiglein, Nucl. Phys. B 690 (2004) 62; S. Heinemeyer, 
D. Stockinger and G. Weiglein, Nucl. Phys. B 699 (2004) 103; Tai-Fu Feng, Tao Huang, Xue-Qian Li, Xin-Min Zhang, Shu-Min Zhao, Phys. Rev. D 68 (2003) 016004.

[12] Hai-Bin Zhang, Tai-Fu Feng, Shu-Min Zhao, Tie-Jun Gao, Nucl. Phys. B 873 (2013) 300; Shu-Min Zhao, Tai-Fu Feng, Tong Li, Xue-Qian Li, Ke-Sheng Sun, Mod. Phys. Lett. A 27 (2012) 1250045; Xiu-Yi Yang, Tai-Fu Feng, Phys. Lett. B 675 (2009) 43.

[13] A. Pilaftsis, Phys. Rev. D 58, (1998) 096010; M. Carena, J. Ellis, A. Pilaftsis and C. E. M. Wagner, Nucl. Phys. B 586, (2000) 92; Tai-Fu Feng, Lin Sun, Xiu-Yi Yang, Nucl. Phys. B 800 (2008) 221; Tai-Fu Feng, Lin Sun, Xiu-Yi Yang, Phys. Rev. D 77 (2008) 116008.

[14] I. S. Alatrev et. al., Phys. Lett. B 276, (1992) 242; K. F. Smith et. al., Phys. Lett. B 234, (1990) 191.

[15] E. P. Shabalin, Yad. Fiz. 28,(1978) 151; Sov. J. Nucl. Phys. 28,(1978) 75.

[16] J. Ellis, S. Ferrara, and D. V. Nanopoulos, Phys. Lett. B. 114,(1982) 231; W. Buchmuller and D. Wyler, ibid. 121,(1983) 321; J. Polchinski and M. B. Wise, ibid. 125,(1983) 393.

[17] P. Nath, Phys. Rev. Lett. 66, (1991) 2565; Y. Kizukuri and N. Oshimo, Phys. Rev. D. 46,(1992) 3025; 45,(1992) 1806.

[18] T. Ibrahim and P. Nath, Phys. Rev. D 57, (1998) 478; T. Ibrahim and P. Nath, Phys. Rev. D 58, (1998) 019901.

[19] D. Chang, W. Chang and W. Keung, Phys. Rev. D 71, (2005) 076006; G. Giudice and A. Romanino, Phys. Lett. B 634, (2005) 69.

[20] Tai-Fu Feng, Xue-Qian Li, Jukka Maalampi, Xin-min Zhang, Phys. Rev. D 71 (2005) 056005.

[21] J. Dai, H. Dykstra, R.G. Leigh, S. Paban, and D.A. Dicus, Phys. Lett. B 237,(1990) 216; D. A. Dicus, Phys. Rev. D. 41,(1990) 999.

[22] R. Arnowitt, J. Lopez, and D. V. Nanopoulos, Phys. Rev. D. 42, (1990) 2423; R. Arnowitt, M. Duff and K. Stelle, ibid. 43, (1991) 3085.

[23] A. Manohar, and H. Georgi, Nucl. Phys. B. 234,(1984) 189.

[24] A. Arbey, M. Battaglia, A. Djouadi and F. Mahmoudi, JHEP 1209 (2012) 107. 